Revue des patrimoines

$42 \mid 2020$

Imagerie numérique et patrimoine culturel :

représentation et transmission des connaissances

\title{
Les « trois Trésors sacrés » de la monarchie japonaise : un « patrimoine caché »?
}

The " three sacred treasures" of the Japanese monarchy: an " hidden heritage »?

Éric Seizelet

(2) OpenEdition Journals

Édition électronique

URL : http://journals.openedition.org/insitu/28162

DOI : $10.4000 /$ insitu. 28162

ISSN : 1630-7305

Éditeur

Ministère de la Culture

Référence électronique

Éric Seizelet, « Les «trois Trésors sacrés » de la monarchie japonaise : un «patrimoine caché »? », In Situ [En ligne], 42 | 2020, mis en ligne le 12 juin 2020, consulté le 12 janvier 2021. URL : http:// journals.openedition.org/insitu/28162 ; DOI : https://doi.org/10.4000/insitu.28162

Ce document a été généré automatiquement le 12 janvier 2021.

\section{(i) $\odot$

In Situ Revues des patrimoines est mis à disposition selon les termes de la licence Creative Commons Attribution - Pas d'Utilisation Commerciale - Pas de Modification 4.0 International. 


\section{Les «trois Trésors sacrés » de la monarchie japonaise : un " patrimoine caché »?}

The " three sacred treasures " of the Japanese monarchy: an " hidden

heritage »?

Éric Seizelet

«Les trois Trésors sacrés de la monarchie sont consubstantiels à l'existence du trône et se tiennent toujours à ses côtés. Il va d'ailleurs sans dire qu'ils en sont le symbole même ». Cette déclaration du ministre d'État Tokujirô Kanamori (1886-1959), qui avait la lourde charge de présenter et de défendre devant la Diète le nouveau projet de loi fondamentale, qui allait supplanter la charte de Meiji de 1889, signifie que quels que soient les changements opérés dans la position et les pouvoirs de l'empereur dans les nouvelles institutions, ils n'affectent en rien le statut et le rôle des regalia ${ }^{1}$. Bien des décennies plus tard, le $1^{\mathrm{er}}$ mai 2019 s'est déroulée la première des grandes cérémonies qui ponctuent la succession au trône du Chrysanthème : la cérémonie de remise des regalia, par laquelle les insignes de la monarchie ont été solennellement transférés au nouvel empereur, le prince héritier Naruhito. Parmi eux, les "trois Trésors sacrés", sanshu no jingi, - le Miroir, l'Épée et le Joyau (fig. 1) - transmis d'âge en âge au nouveau tennô, et longtemps présentés à la fois comme des symboles de légitimité et des palladia ${ }^{2}$. 


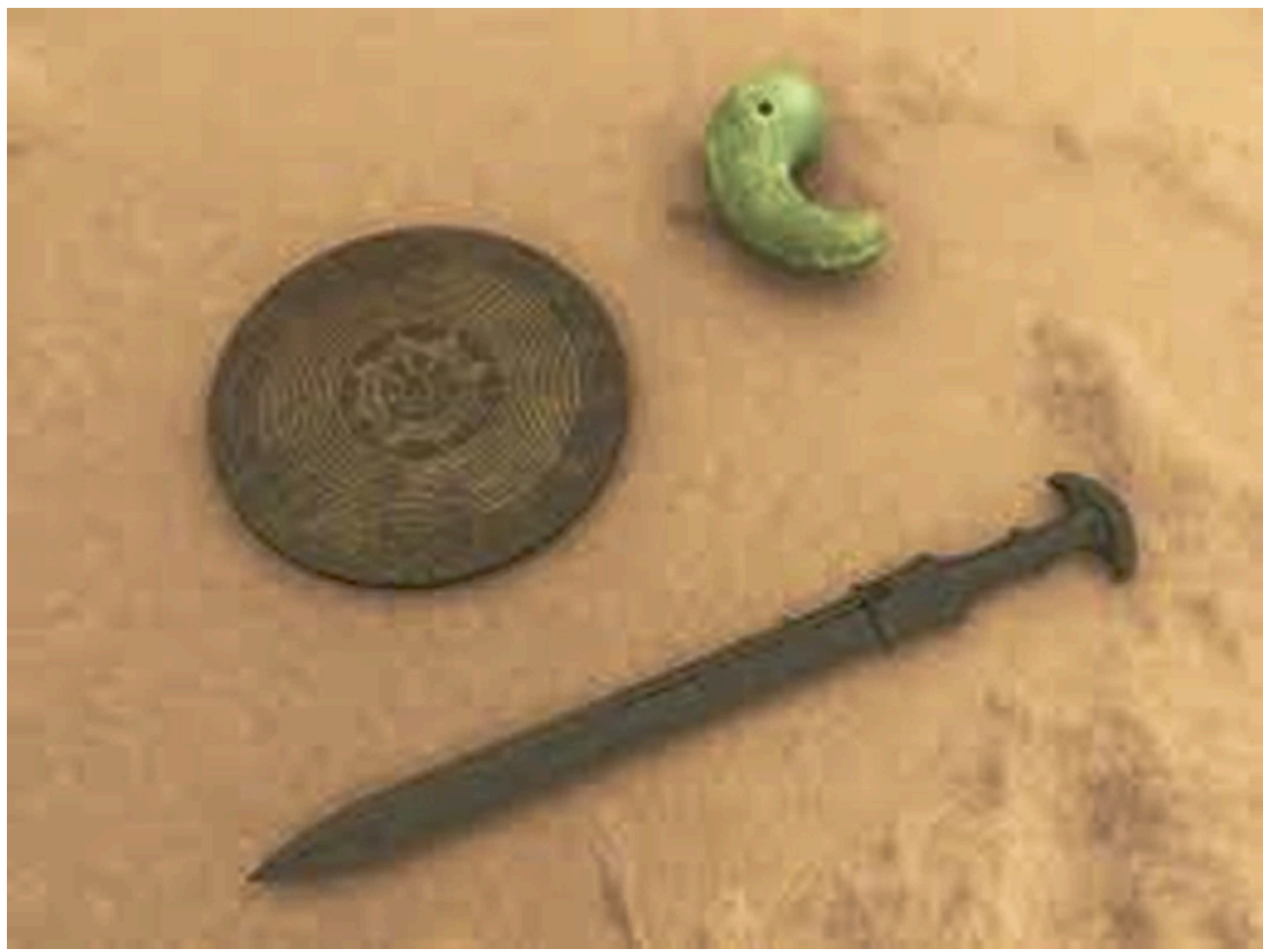

Aspect supposé des regalia japonais.

Source : https://www.excite.co.jp/news/article/BestTimes_8182/

2 L'occasion de rappeler que si ces insignes ont une longue tradition, leur inclusion dans la catégorie des "biens culturels ", bunkazai, reste un sujet de débat dans l'Archipel autour de la singularité de leur histoire, de leur caractérisation patrimoniale, et des cérémonies entourant leur transmission.

\section{Les regalia japonais : une histoire singulière}

3 Singulière, d'abord, du fait de leur attribution mythologique. Ils sont censés avoir été donnés par la déesse solaire Amaterasu-Ômi-Kami, la divinité tutélaire de la famille impériale, à son petit-fils Ninigi-no-Mikoto, lorsque celle-ci lui a ordonné de descendre du Ciel pour régner, lui et ses descendants, à jamais, sur le Japon. Telle est l'origine des regalia, du moins, d'après le récit qu'en font les premières chroniques historiques japonaises datant du début $\mathrm{du} \mathrm{vIII}^{\mathrm{e}}$ siècle de notre ère, retraçant la création de l'Archipel et l'origine de la monarchie.

4 Singulière, aussi, car les insignes actuels conservés au Palais impérial actuel ne sont pas tous des « originaux » mais des répliques hiérophaniques, katashiro. Le Palais impérial, et plus particulièrement le salon Kenji no $\mathrm{Ma}$, abriterait l'original du Joyau, mais le Miroir et l'Épée sont des répliques d'objets abrités par le grand sanctuaire d'Ise et le grand sanctuaire d'Atsuta (Nagoya), se trouvant respectivement au sanctuaire Kashikodokoro du Palais et au salon précité. Cette dénomination fait elle-même problème, car il n'existe aucune traçabilité scientifique de ces objets permettant d'assurer que les insignes abrités par ces deux grands sanctuaires peuvent être identifiés comme authentiques. Et ils n'ont pas été épargnés par les vicissitudes de 
l'histoire : les chroniques font état, à plusieurs reprises, de vols, de déprédations à la suite d'incendies ou de pertes. Le 25 avril 1185, par exemple, les regalia du Palais auraient sombré lors de la bataille navale de Dan no ura opposant le clan des Taïra aux Minamoto, et seuls le Joyau et le Miroir auraient été récupérés. Il n'en demeure pas moins qu'ils restent investis, par le mystère entourant leur origine, d'une charge symbolique importante: leur valeur ne tient pas tant à leur authenticité, à leur esthétique supposée ou aux techniques utilisées pour leur fabrication qu'à leur lien consubstantiel avec la monarchie dont elle exprime à la fois, l'ancienneté, la sacralité, la pérennité, et aux représentations morales, voire sexuelles, qui leur sont associées ${ }^{3}$. C'est la raison pour laquelle ces répliques ne peuvent être considérées ni comme des reliques ni comme de simples réceptacles profanes dépourvus d'âme.

5 Singulière aussi par certains épisodes restés gravés dans la mémoire collective : le 11 février 1889, le jour même de la promulgation de la constitution de l'Empire du Grand Japon, le ministre de l'Éducation MORI Arinori (1847-1889), l'une des «Lumières » du Japon de Meiji, fut mortellement blessé pour avoir violé le strict protocole entourant les visites au grand sanctuaire d'Ise. Les historiens et archéologues ont pu néanmoins établir que ces trois objets ne sont pas le monopole de la Maison impériale : on les retrouve dans des sanctuaires shintô et des temples bouddhistes, dans des sépultures et tertres anciens, kofun, de personnages importants au Japon comme en Corée, ou sous forme d'objets de culte pendant la période Yayoi (vers -400 av. J.-C. $\sim+350$ ap. J.-C.), et en Chine en tant que puissants talismans taoïstes ${ }^{4}$. Mais on n'a jamais pu établir leur présence dans les tumulus impériaux, l'agence de la Maison impériale restant hostile aux fouilles approfondies sur ces sites, au motif qu'ils abritent les tombes des ancêtres de la famille impériale auxquels est encore rendu un culte par des cérémonies particulières ${ }^{5}$.

6 Singulière, enfin, parce qu'en raison de leur nature particulière et des effets magiques qui leur sont attribués, ils n'ont fait l'objet d'aucune ostension ou exposition ${ }^{6}$. Il est même douteux que les empereurs aient pu les contempler ${ }^{7}$. C'est ainsi qu'à la différence des monarques occidentaux exhibant avec faste les insignes de leur position lors de certains événements de leur règne, telles les cérémonies du couronnement ou du sacre, les souverains japonais, même dans les photos officielles, n'ont jamais été représentés arborant les insignes de la monarchie. Lors de la cérémonie d'accession au trône, les regalia sont présentés au nouvel empereur par les chambellans, mais enfermés dans deux coffrets ${ }^{8}$. Certes, la coutume veut que les déplacements impériaux hors du palais soient accompagnés de la translation des regalia - de deux d'entre eux en pratique, l'Épée et le Joyau - mais outre le fait que cette translation n'existe guère plus que lors des pèlerinages de l'empereur au grand sanctuaire d'Ise, pour des raisons de sécurité, ils sont toujours enfermés dans des coffrets spéciaux (fig. 2). 
Figure 2

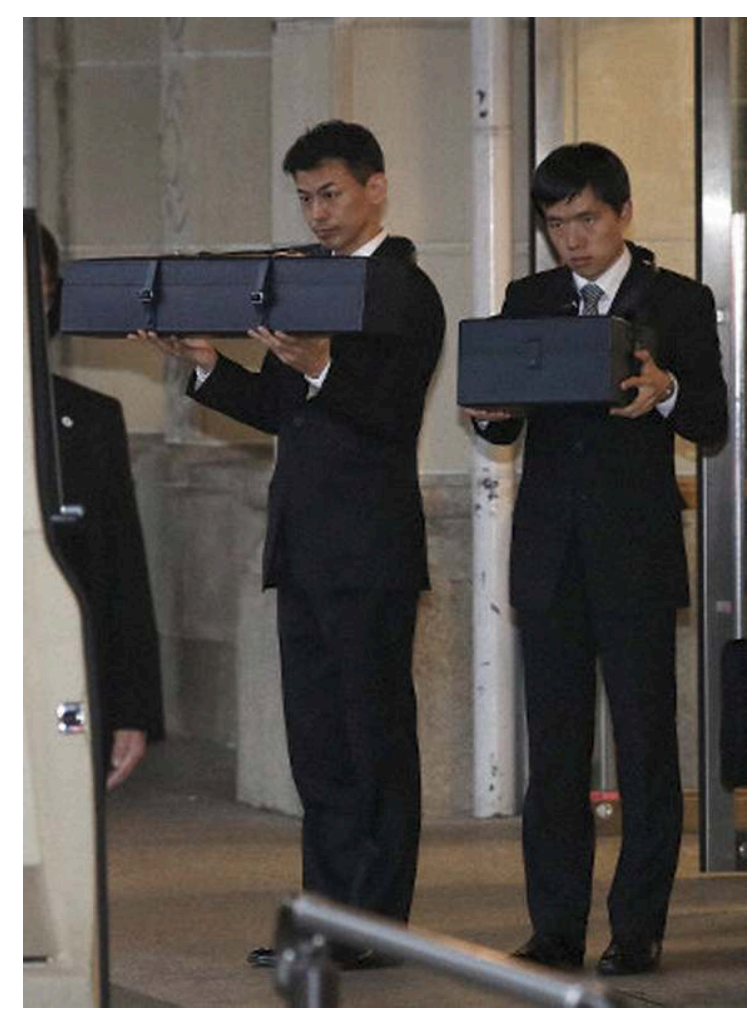

Arrivée des regalia en gare de Ujidayama à l'occasion de la dernière visite du couple impérial au grand sanctuaire de Ise pour rapporter à la déesse solaire la prochaine abdication de l'empereur.

Source : Mainichi shinbun, 17 avril 2019.

De même, lors de la reconstruction rituelle du grand sanctuaire d'Ise tous les vingt ans, le Miroir fait l'objet d'une translation dans les nouveaux bâtiments au cours d'une procession nocturne, mais avec des précautions particulières de manipulation, à l'abri de tout regard. À la Diète, les directeurs de l'agence de la Maison impériale ont confirmé qu'ils n'ont jamais vérifié le contenu des coffrets9. D'où une question lancinante, comment protéger des biens soustraits à la vue du commun et dont on ne possède aucune représentation, aucun dessin fiables?

\section{Les regalia : un patrimoine non classé}

Hétérodoxes du point de vue historique, les regalia, le sont aussi du point de vue juridique. Considérés comme indissociables du trône impérial, ils ne figurent pourtant ni dans la constitution, ni dans le code actuel de la Maison impériale. Un silence justifié par le fait que la nouvelle loi fondamentale reconnaissant la liberté de croyance et de pensée, il n'avait pas été jugé approprié de les intégrer dans les textes fondateurs d'un régime sécularisé ${ }^{10}$. Selon l'interprétation la plus communément admise, les insignes de la monarchie font partie des biens meubles et immeubles qui relèvent de la catégorie des « objets historiques qui doivent être transmis avec le trône », en vertu de l'article 7 de la loi sur l'économie de la Maison impériale de 1947. Il faut entendre par là les "biens qui sont depuis longtemps en possession de la Maison impériale », ce qui signifie, d'une part, que la durée de détention est un élément important de cette qualification et que, d'autre part, les biens les plus récents entrés dans le patrimoine de 
la Maison impériale n'en font pas partie ${ }^{11}$. Toutefois, il n'existe aucune liste préétablie de tels biens. Si certains d'entre eux, d'ailleurs parmi les plus communément cités comme les regalia et les trois sanctuaires du Palais pour la célébration des rites de la Maison impériale, y figurent «naturellement», ce n'est guère - à notre connaissance - qu'au moment de la succession qu'un inventaire détaillé, gyôbutsu chôsho, mais non publié, du patrimoine de la Maison impériale est effectué, notamment pour des raisons fiscales. Cet inventaire est un "document administratif » et comme tel, relève, sous conditions, de la procédure d'accès aux informations administratives dans le cadre d'une loi entrée en vigueur le $1^{\mathrm{er}}$ avril 2001.

9 Font-ils partie du patrimoine artistique et culturel japonais? Pour les élites conservatrices du pays, la réponse positive ne fait aucun doute. Mais cette intégration ne résulte pas d'une formalisation à travers les procédures de protection du patrimoine national ${ }^{12}$. L'article 2 de la loi révisée de 1950 sur la protection des biens culturels définit de la façon suivante les «biens culturels », bunkazai : «l'ensemble des productions culturelles, telles que les constructions, les peintures, les sculptures, les objets d'art et d'artisanat, les textes anciens possédant une grande valeur artistique ou historique pour notre pays. " La catégorie des «biens culturels importants », jûyô bunkazai désigne ceux de ces biens qui font l'objet d'une décision de classement par le ministre de l'Éducation. Celle des "Trésors nationaux », kokuhô, désigne ceux de ces biens qui sont classés comme tels par le ministre « en raison de leur valeur du point de vue du patrimoine culturel mondial et qui présentent, en tant que trésors de la nation, un caractère rare et exceptionnel ». Les regalia auraient-ils pu figurer dans la liste des Trésors nationaux ? Force est de constater qu'en l'état actuel du débat, les insignes monarchiques échappent aux politiques publiques de protection et de conservation du patrimoine : ils ne figurent ni au classement des "Trésors nationaux", ni à celui des "biens culturels importants", ni à celui de l'inventaire des biens culturels inscrits. Ils ne sont pas assurés contre le vol ou la destruction accidentelle, puisqu'ils n'ont fait l'objet d'aucune expertise. Cette exclusion du classement ne concerne pas seulement les regalia mais l'ensemble du patrimoine appartenant à la Maison impériale, kôshitsu zaisan, à l'exception du bâtiment du Shôsô-in (Nara) ${ }^{13}$, classé « Trésor national » en 1997. L'agence de la Maison impériale s'est bornée à indiquer que « le maintien et la gestion » de ces biens transmis lors de la succession au trône sont assurés sur les crédits de la Cour interne, naiteihi, par les chambellans impériaux auxquels revient la responsabilité d'assurer leur sécurité et leur évacuation en cas de problème. Mais, outre le fait que les chambellans qui assurent le service quotidien des membres de la famille impériale ne sont pas des professionnels de la conservation, l'agence de la Maison impériale n'a jamais précisé, concrètement, en quoi consistait le travail de " maintien et de gestion » effectué par eux. Officiellement, selon le langage utilisé par l'agence de la Maison impériale, les regalia ne sont pas conservés au Palais ou dans les grands sanctuaires. Ils $\mathrm{y}$ "reposent", hoan, ou anchi. La question néanmoins du classement des «objets historiques » a parfois été évoquée à la Diète au motif que ces «trésors » appartiennent autant à la nation tout entière qu'à la Maison impériale, soit au titre de "Trésor national ", soit dans le cadre d'une catégorie sui generis qu'il conviendrait au ministère de l'Éducation d'établir. Toutefois, l'agence de la Maison impériale ne s'y est pas montrée favorable parce qu'il existait déjà des vestiges, objets et documents, placés sous le contrôle de l'agence dont l'importance historique était indéniable mais qui ne figuraient pas pour autant à l'inventaire des pièces classées «biens culturels 
importants", ou "Trésors nationaux » et que l'identification des regalia en tant qu'« objets historiques » suffisait à les protéger ${ }^{14}$.

Reste la question du droit de propriété : les regalia appartiennent-ils au patrimoine de l'État ou à l'empereur? Sur ce point, la réponse est claire: les regalia, comme les " objets historiques ", ne font partie ni du patrimoine de l'État, ni du domaine public, notamment en raison de leur connotation religieuse ${ }^{15}$. Ils appartiennent donc au monarque. Mais la question se pose néanmoins de la nature, publique ou privée, de ce droit de propriété. Le directeur de l'agence de la Maison impériale USAMI Takeshi (1903-1991) avait confirmé devant la Diète, dans un premier temps, que même si l'article 7 de la loi sur l'économie de la Maison impériale ne précise pas la nature des " objets historiques" qui doivent être remis à l'héritier du trône au moment de la succession, les trois insignes de la monarchie en font partie ${ }^{16}$. Ce sont donc des «biens héréditaires " qui relèvent du droit de "propriété privée ", shiyû, du monarque ${ }^{17}$. Ces insignes ne concernent pas seulement ceux qui se trouvent au Palais impérial, mais également le Miroir et l'Épée honorés dans les grands sanctuaires d'Ise et d'Atsuta qui n'en sont ni les dépositaires ni les propriétaires attitrés. On mesure, à l'aune de cette prise de position, l'ambiguïté du statut des regalia japonais. Comment reconnaître un droit de propriété privée du monarque sur ces insignes liés au trône? D'autant que la loi stipule que l'empereur a l'obligation de transmettre les objets dont il s'agit au prince héritier. C'est donc qu'ils sont frappés d'inaliénabilité et d'imprescriptibilité puisque l'empereur ne peut en disposer librement. Il faut donc considérer que l'empereur n'en est pas propriétaire au sens du droit privé, mais dépositaire en tant que chef de la Maison impériale et au titre de symbole constitutionnel de l'État et de l'unité du peuple, et que si droit de propriété il y a, il s'agit d'une propriété publique liée au trône et donc à la dévolution ${ }^{18}$.

\section{Un statut fiscal exorbitant du droit commun}

11 Sur le plan fiscal, seuls les « objets historiques qui doivent être transmis avec le trône impérial » sont exemptés des droits de succession, conformément à l'article 12 alinéa 1 du Code successoral. Pour trois motifs : cette transmission concerne des biens attachés à la dévolution et à la position constitutionnelle du trône; les biens ne peuvent être aliénés et n'ont donc pas de valeur marchande ; il s'agit d'une faveur spéciale concédée par l'État à la Maison impériale. La direction législative du Cabinet a notamment indiqué que l'intention du législateur, lorsque la loi sur l'économie de la Maison impériale est venue en discussion à la Diète, a été d'éviter que ces " objets historiques » ne puissent faire l'objet d'un partage, conformément au droit commun de la succession $^{19}$. Une difficulté est néanmoins apparue avec la succession actuelle: le prince impérial NARUHITO a accédé au trône non à la suite du décès de son père AKIHITO mais de son abdication. Juridiquement, la transmission des regalia ne s'effectuait pas par succession mais par donation entre vifs normalement soumise à l'impôt ${ }^{20}$. Encore aurait-il fallu pour cela procéder à l'évaluation de ces biens pour en déterminer l'assiette. Le gouvernement a donc décidé d'inscrire dans la loi spéciale $\mathrm{n}^{\circ} 63$ du 16 juin 2017 adoptée par le Parlement japonais autorisant l'abdication de l'empereur à titre exceptionnel une disposition additionnelle $\mathrm{n}^{\circ} 7$ garantissant l'exemption fiscale des droits de donation. À la fois pour ne pas introduire de rupture par rapport au précédent de 1989, et parce que l'évaluation des regalia soulevait des 
obstacles insurmontables. Les " objets historiques » ont échappé au régime de taxation des donations et des successions ainsi qu'aux dispositions constitutionnelles de l'article 8 qui prévoient que toute transaction en provenance ou à destination de la Maison impériale doit être autorisée par la Diète, dans la mesure où la donation est intervenue à l'intérieur même de la Maison impériale. En revanche, l'ensemble des biens culturels et des œuvres d'art détenus en propre, Gyôbutsu, par l'empereur précédent, et qui ne figurent pas dans cette nomenclature, sont taxables : l'exonération ne couvre pas les « objets historiques » qui ne sont pas directement liés à la dévolution. En 1989, lors de la succession de l'empereur Hirohito, ces «objets » ont représenté quelque 580 items (insignes monarchiques, sanctuaires du Palais, portraits de membres de la famille impériale, autographes et calligraphies, parures et vêtements, paravents, bibliothèque de Higashiyama, sabres précieux et cérémoniels notamment) sur les 4600 faisant l'objet de l'inventaire successoral ${ }^{21}$. En d'autres termes, tout est affaire d'assiette : plus nombreuses seront les collections impériales cataloguées dans le champ d'application de l'article 7 de la loi sur l'économie de la Maison impériale et plus l'assiette de l'impôt sera réduite. Enfin, les «objets historiques " ne peuvent être donnés à l'État puisque c'est l'héritier du trône qui doit en prendre possession ${ }^{22}$.

\section{La transmission des insignes monarchiques : une cérémonie publique?}

La cérémonie de Remise des insignes monarchiques, Kenjitô keisho no gi, fut très courte (moins d'une dizaine de minutes): dans la grande salle du Palais Matsu no Ma, les chambellans ont placé solennellement, sur des présentoirs devant l'empereur, une série de coffrets contenant, outre les regalia, le sceau de l'État et le sceau impérial (fig. 3).

Figure 3

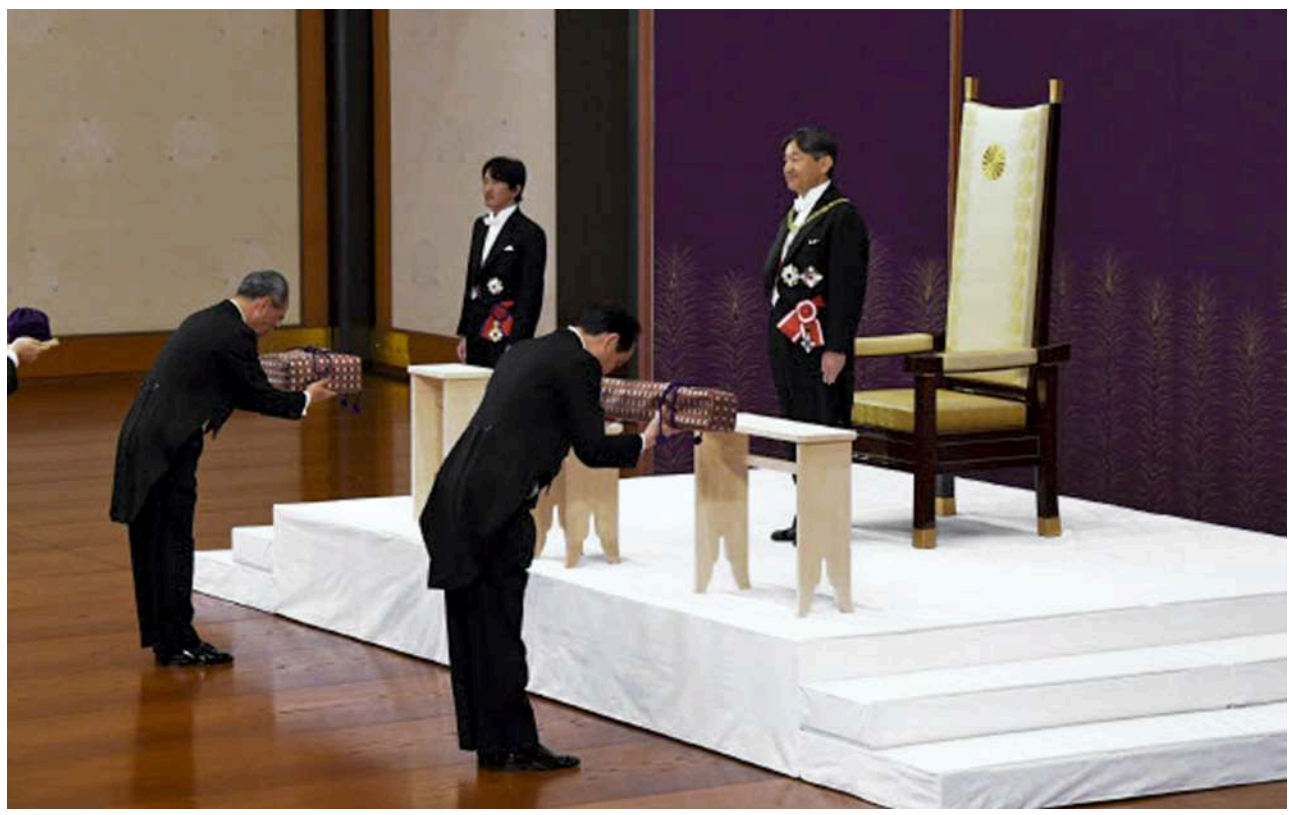

Présentation solennelle des regalia au nouvel empereur.

Source : Nikkei shinbun, $7^{\text {er }}$ mai 2019. 
13 Elle s'est déroulée en présence des plus hauts représentants de l'État et des membres mâles adultes de la famille impériale. Cette cérémonie, laïcisée à dessein - elle ne portait plus le nom de togyô no gi comme avant 1945, et la remise des sceaux avait été ajoutée - soulève néanmoins un certain nombre de questions.

14 La première est l'exclusion des femmes appartenant à la famille impériale, y compris la nouvelle impératrice. Elle se justifie, en théorie du moins, par le fait que le Code de la Maison impériale de 1947 confirme le principe exclusif de patrilinéarité dans les mécanismes de succession au trône et que, conformément à une décision gouvernementale prise le 17 janvier 2019, les autorités, en s'en tenant aux précédents, n'ont pas voulu relancer les discussions sur la succession au trône par les femmes ${ }^{23}$. Elle apparaît, néanmoins, en contradiction avec la politique de promotion de la place de la femme dans la société japonaise mise en avant par le cabinet Abe depuis son entrée en fonction, inutilement discriminatoire puisque la seule femme membre du gouvernement, Mme KATAYAMA Satsuki, ministre déléguée en charge de la Réactivation des régions et de la Promotion de l'égalité des genres, a pu y assister, et juridiquement contestable puisque les femmes peuvent être régentes. Elle signifie en tout cas que le principe d'égalité inscrit à l'article 14 de la loi fondamentale n'a pas encore totalement franchi les douves du Palais impérial.

La deuxième est l'intention gouvernementale de « se conformer fondamentalement aux précédents ». Ni la constitution, ni le Code de la Maison impériale ne renferment de dispositions particulières sur l'organisation et le type de cérémonies organisées lors d'un changement de règne. Comme en 1989, le gouvernement s'est calqué sur une ordonnance impériale de 1909 sur la dévolution, tacitement abrogée en 1947 lors de l'entrée en vigueur de l'actuelle loi fondamentale, mais dont les dispositions subsistent néanmoins à l'état coutumier. Or, en dépit des précautions sémantiques précitées, le recours aux " précédents ", sanctifié au nom du respect de la "tradition », ne fait-il pas problème puisque la «tradition » en question fait référence au Japon impérial, dans lequel la position, le statut et les pouvoirs de l'empereur étaient fort différents de ceux qui lui ont été conférés sous le Japon démocratique?

La troisième concerne le principe de séparation de l'État et de la religion. En classant, le 3 avril 2018, la cérémonie de transmission des insignes de la monarchie parmi la catégorie des "actes en matière de représentation de l'État ", c'est-à-dire des actes constitutionnels accomplis par l'empereur sur avis et approbation du Cabinet, le gouvernement n'aurait pas voulu seulement donner une tournure officielle aux cérémonies de transition mais avaliser des traditions mythologiques qui ont été au cœur du shintoïsme d'État. La critique ne concerne pas, il faut le souligner, le principe même de ce type de cérémonie dans la mesure où ces insignes font partie des « objets historiques » visés par la loi précitée de 1947. Et le caractère religieux des regalia ne fait guère de doute. Si la cérémonie se déroule dans un espace neutre, le Palais impérial, et ne fait intervenir aucun personnel ou rite religieux, la question demeure du soutien officiel et de la prise en charge par l'État de l'organisation de cette cérémonie. Les partisans de la laïcité à la japonaise estiment qu'en vertu du principe de neutralité religieuse de l'État, cette cérémonie aurait dû avoir un caractère privé. Certes, le Code de la Maison impériale prévoit bien une cérémonie de l'Avènement, Sokui no rei, mais il ne dit rien des rites d'accession, dont le transfert des regalia. En organisant un régime officiel de transition monarchique qui, au nom de la tradition, reproduit la dualité des cérémonies qui avaient cours avant 1945, le gouvernement n'aurait fait que céder aux 
pressions du lobby shintô, afin de promouvoir une conception particulière de la conception de "symbole de l'État et de l'unité du peuple", dans laquelle les adeptes d'autres religions telles que le christianisme et le bouddhisme peuvent difficilement se reconnaitre ${ }^{24}$. Pour d'autres, en revanche, le Code de la Maison impériale ne fait que distinguer l'existence d'une cérémonie particulière et n'entend pas prohiber l'existence d'autres cérémonies; le fait que la transition monarchique comporte deux types de cérémonies distinctes est bien antérieur à la mise en place du shintoïsme d'État à partir de l'ère Meiji ; considérer que la remise des regalia devrait être privatisée, au même titre que les autres "objets historiques", est absurde puisqu'ils sont liés au trône impérial, kôi, qui est une institution de l'État reconnue par la loi fondamentale ; enfin, il est question d' "objets ", mono, une qualification neutre qui n'a aucune connotation religieuse. Rien ne devrait donc empêcher que leur transmission prenne la forme publique d'un acte en matière de représentation de l'État. Le fond de la question n'est donc pas de savoir si le gouvernement est autorisé ou non à organiser des cérémonies de transition mais de déterminer si l'organisation par l'État de ces cérémonies satisfait aux contraintes constitutionnelles, et notamment au principe de séparation de l'État et de la religion ${ }^{25}$.

\section{Conclusion}

17 La catégorisation des « objets historiques » illustre le double standard qui préside à la protection par l'État du patrimoine national au Japon: d'un côté, des biens culturels répertoriés et protégés comme tels. De l'autre, des biens intimement liés à la Maison impériale, non officiellement répertoriés, et conservés selon des procédures internes échappant non seulement à tout contrôle démocratique mais aussi à toute expertise fiscale et artistique externe. Certes, les regalia et avec eux "les objets historiques ", renvoient à une conception " continuiste » du patrimoine japonais, couvrant « tous les biens, tous les trésors du passé ", à la croisée du "fait religieux» et du "fait monarchique ", indépendamment de l'identité de l'entité qui les détient ${ }^{26}$. L'intention de patrimonialisation, c'est-à-dire la volonté des acteurs, en l'espèce l'agence de la Maison impériale, de conférer à ces biens des propriétés et des valeurs dignes d'être transmises aux générations futures est également manifeste. Les regalia constituent, en ce sens, de véritables artefacts. Mais dotés d'une mémoire particulière qui fait l'objet de débats dans la société japonaise. Il reste que les regalia sont entourés d'un épais voile de mystère puisque ni la définition des « objets historiques ", dont il s'agit, ni leur mode de transmission n'ont été juridiquement précisés. Paradoxalement, le gouvernement, en officialisant la remise des regalia au nouveau monarque, a cherché «à manifester devant la nation la réalité de l'accession au trône ». Mais ce souci de transparence n'est pas allé jusqu'à dévoiler au public les insignes en question. Pour lever les incertitudes sur leur apparence, n'aurait-il pas suffi que l'agence de la Maison impériale, de concert avec la famille impériale et le gouvernement, décide de les exposer, à l'occasion d'un changement de règne par exemple? Les autorités d'Occupation avaient envisagé un moment de le faire après 1945 pour couper court à la « divinité » impériale et éradiquer le shintoïsme d'État, mais sans succès. Au fond, il en va peut-être des regalia japonais comme d'autres objets mythiques tels que l'Arche d'alliance, prétendument conservée à Aksoum en Éthiopie, ou la Sainte Lance qui aurait transpercé le flanc du Christ sur la 
croix : des objets de croyance et de vénération, qui rendraient superfétatoire et vaine toute interrogation sur leur véracité et leur authenticité.

\section{NOTES}

1. Chambre des représentants, Commission sur la révision de la constitution de l'Empire, 12 juillet $1946, \mathrm{n}^{\circ} 11$.

2. Palladium : entité concrète ou abstraite assurant la sauvegarde ou la survie d'une collectivité, d'une institution, d'une valeur (TLF). Nous renvoyons à notre étude : SEIZELET Éric, « Les trois Trésors sacrés et la symbolique impériale au Japon. Passé et présent ", Bulletin du Centre de recherche du château de Versailles, 2005 [en ligne], https://journals.openedition.org/crcv/408 [lien valide en juin 2020] ; LAHOURNAT Florence, « Matérialiser l'intangible. Étude des objets supports de la divinité dans le Japon ancien ", thèse de doctorat en langues, littératures et sciences sociales orientales (Études japonaises) soutenur à l'Inalco, Paris, 2007 ; KADOYA Atsushi, « Myths, rites and icons ", in SCHEID Bernhard \& TEEUWEN Mark (dir.), The Culture of secrecy in Japanese religion, New York, Routledge, 2006, p. 269-283.

3. Selon le lettré néo-confucianiste KUMAZAWA Banzan (1619-1691), l'Épée symboliserait le courage, le Miroir la connaissance, et le Joyau l'humanité. D'après d'autres interprétations, les regalia seraient des symboles de fertilité et de fécondité : l'Épée représenterait le sexe masculin, le Miroir le sexe féminin, et le Joyau le fœtus issu de leur union. Sur l'évolution des interprétations au cours de l'histoire, voir TAKENADA Shinsuke. "Sanshu no jingi no shôchôteki imi no kaishaku wo megutte - shinboru to shite no kagami, tsurugi, tama ni sonawaru tagisei ni tsuite [À propos de l'interprétation du sens symbolique attribué aux trois trésors sacrés. Le caractère polysémique du Miroir, de l'Épée et du Joyau] ", Gengo to bunmei : ronshû, n 12,2014 , p. 131-151.

4. SEIDEL Anna, "Kokuho. Note à propos du terme "Trésor national" en Chine et au Japon ", Bulletin de l'École francaise d'Extrême-Orient, n 69, 1981, p. 229-261, [disponible en ligne] https:// www.persee.fr/doc/befeo_0336-1519_1981_num_69_1_3364?q=trésor+seidel+anna [lien valide en juin 2020].

5. Sur la question, voir: AGATA Yukio, "Shiseki to shite ryôbo no kenpô mondai [Questions constitutionnelles relatives aux mausolées impériaux en tant que vestiges historiques] », Ôtsuma daigaku kiyô bunkei, vol. 29, 1997, p. 289-299.

6. Il en est de même des reportages : le grand sanctuaire d'Atsuta, par exemple, oppose toujours une fin de non-recevoir aux organes de presse souhaitant avoir accès à l'Épée.

7. WATANABE Daimon, «Sanshu no jingi no shinpi. Mita mono ni ha tenbatsu ga kudaru. Tennô saemo mitakoto no dekinai. Himerareta chikara [Le mystère des trois Trésors sacrés: la malédiction qui frappe ceux qui les ont vus. Même l'empereur ne peut les voir. Une force cachée] », Rekishi dokuhon, vol. 55, n 8, 2010, p. 194-199.

8. Le Miroir n'est pas présenté lors de cette cérémonie d'accession. Il reste au Kashikodokoro mais un émissaire impérial est dépêché auprès du sanctuaire pour rapporter à la déesse solaire l'avènement du nouveau monarque.

9. USAMI Takeshi, Chambre des représentants, Commission du Cabinet, 30 mars 1962, nº 6. 
10. FUTURA Yoshinori, président de la Commission de révision du Code de la Maison impériale, Chambre des pairs, séance plénière, 11 décembre 1946, n 11 ; KANAMORI Tokujirô, Chambre des pairs, Commission de révision du Code de la Maison impériale, 17 décembre 1946, nº 2.

11. La mise en place de cette loi s'est faite en étroite concertation avec les autorités d'occupation. L'article 7 a fait l'objet de discussions bilatérales entre le 19 novembre et le 2 décembre 1946, d'où il ressort que le General Headquarters of the Allied Forces a admis l'inclusion des regalia au nombre des objets précités : ASHIBE Nobuyoshi \& TAKAMI Katsutoshi, Nihon rippô shiryô zenshû 7 kôshitsu keizaihô [Compendium de législation japonaise. Vol. 7: La loi sur l'économie de la Maison impériale], Tokyo, Shinzansha, 2002, p. 655. Sur le moment de l'inventaire, voir MIYAO Iwao, vicedirecteur de l'agence de la Maison impériale, Chambre des conseillers, Commission de l'administration locale, 29 mars 1990, $\mathrm{n}^{\circ} 2$. Sur le souhait de certains parlementaires que l'inventaire des biens précités soit communiqué au public, et sur l'impossibilité d'obtenir une réponse claire des pouvoirs publics quant à leur nomenclature, voir ISAYAMA Hiroshi, Parti communiste, Ibid.

12. Sur la protection du patrimoine au Japon et son histoire, voir : BOURDIER Marc, « Le mythe et l'industrie ou la protection du patrimoine culturel au Japon ", Genèses. Sciences sociales et histoire, $\mathrm{n}^{\circ} 11, \quad 1993$, p. 82-110, [disponible en ligne] https://www.persee.fr/doc/ genes_1155-3219_1993_num_11_1_1172 [lien valide en juin 2020] ; POTTIER Christian, «Japon. Note sur la protection patrimoniale au Japon ", Bulletin de l'École française d'Extrême-Orient, $\mathrm{n}^{\circ} 82$, 1995, p.339-351, [disponible en ligne] https://www.persee.fr/doc/ befeo_0336-1519_1995_num_82_1_2890 [lien valide en juin 2020]; ALASSIMONE Catherine, "Protection du patrimoine intangible et politique culturelle au Japon ", thèse de doctorat en sciences de l'information et de la communication soutenue à l'université Bordeaux 3, Bordeaux, 1999 ; MARQUET Christophe, «Le Japon moderne face à son patrimoine artistique », Cipango, numéro hors-série, "Mutations de la conscience dans le Japon moderne », 2002, p. 243-304 ; DEMOULE Jean-Pierre \& SOUYRI Pierre-François (dir.), Archéologie et patrimoine au Japon, Paris, Éditions de la Maison des sciences de l'homme, 2008; INADA Takashi, "L'évolution de la protection du patrimoine au Japon depuis 1950: sa place dans la production des identités régionales ", Ebisu, n52, 2015, p. 21-46, [disponible en ligne] https://journals.openedition.org/ ebisu/1576 [lien valide en juin 2020].

13. Construit au $\mathrm{VIII}^{\mathrm{e}}$ siècle de notre ère, il abrite des collections impériales - 9000 objets environ - qui ne sont montrées au public qu'une fois par an et qui n'ont jamais été exposées à l'étranger.

14. FUKUHARA Kunihiko, directeur du bureau de la Commission de protection des biens culturels du ministère de l'Éducation, URYÛ Nobuyoshi, vice-directeur de l'agence de la Maison impériale, Chambre des représentants, Commission du Cabinet, 3 avril 1968, n $8 ; 23$ avril 1968, nº 19.

15. KANAMORI Tokujirô. Chambre des pairs, Commission spéciale sur le projet de loi relatif à l'économie de la Maison impériale, 21 décembre 1946, nº 1. Ils ne relèvent pas non plus des biens et patrimoine de la Maison impériale qui appartiennent à l'État en vertu de la loi fondamentale (art. 8 et art. 88).

16. La liste des " objets historiques » ainsi transmis est décidée par accord entre l'agence de la Maison impériale, la direction législative du Cabinet, «en concertation avec les organismes concernés », mais sur la base de critères qui ne sont pas rendus publics. Il n'existe pas d'organe idoine chargé de procéder à l'identification de ces biens, contrairement à ce qui avait été un moment envisagé à la Diète : KANAMORI Tokujirô. Chambre des pairs, Commission spéciale sur le projet de loi sur l'économie de la Maison impériale, 22 décembre 1946, $\mathrm{n}^{\circ} 2$; également réponse $\mathrm{n}^{\circ} 29$ du 4 juillet 1989 du Premier ministre UNO Sôsuke à la question écrite du député TAKIZAWA Kôsuke, parti démocrate-socialiste, sur la fiscalité des biens de la Maison impériale. 
17. USAMI Takeshi, Chambre des représentants, Commission du Cabinet, 5 mars 1959, $\mathrm{n}^{\circ} 14$; Chambre des représentants, Commission de la loi de règlement budgétaire, 13 novembre 1959, $\mathrm{n}^{\circ} 4$.

18. Premier ministre IKEDA Hayato, réponse $n^{\circ} 2$ du 22 octobre 1960 à la question écrite du député PLD HAMACHI Bunpei sur le Miroir conservé au grand sanctuaire d'Ise.

19. YOSHIKUNI Ichirô, directeur de la Législation du Cabinet ; FUKUHARA Kunihiko, directeur du bureau de la Commission de protection des biens culturels du ministère de l'Éducation, ibid.

20. Shûkan asahi en date du 5 août 2016. Une donation un peu particulière puisqu'il n'y a eu ni manifestation de volonté explicite de Akihito de transférer les «objets historiques » à son fils aîné ni, a fortiori, contrat de donation.

21. MORI Yôhei, Tennôke no zaifu [Le portefeuille de la famille impériale], Tokyo, Chinchôsha, 2003, p. 119 et suiv.

22. MIYAO Iwao, vice-directeur de l'agence de la Maison impériale, Chambre des représentants, Commission des Affaires juridiques, 14 juin 1989, $\mathrm{n}^{\circ} 4$.

23. Asahi shinbun en date du 30 décembre 2017 ; Nikkei shinbun en date du 15 mars 2018.

24. Sur la liaison ancienne et assumée entre la détention des regalia et la sacralité de l'empereur, voir Jinja shinpô des 14 novembre 1974 et 13 janvier 1975.

25. Sur cette controverse, voir : MURAKAMI Shigeyoshi, « Tokuron. Seijika sareta kôi keisho no shûkyôsei [La nature religieuse des cérémonies d'accession au trône et leur politisation] », Rekishi dokuhon, vol. 53, $\mathrm{n}^{\circ}$ 6, 2008, p. 214-220 ; OKUDAÏRA Yasuhiro, Bansei ikkei no kenkyû [Recherches sur la notion de «lignage unique et éternel»], Tokyo, Iwanami gendai bunko, vol.2, 2017, p. 264 ; TAKASHIMA Hiroyuki, «Kôi keisho gishiki no kadai [Problématiques relatives aux cérémonies d'accession au trône] ", Rekishi chiri kyôiku, n 889, 2019, p. 28-33.

26. BABELON Jean-Pierre \& CHASTEL André, La Notion de patrimoine, Paris, Liana Levi, coll. «Opinion », 1994, p. 11, 27 et suiv.

\section{RÉSUMÉS}

Le Miroir, le Joyau et l'Épée constituent les trois insignes traditionnels de la monarchie japonaise transmis à chaque génération d'empereurs. Longtemps associés à une symbolique des vertus prêtées au trône impérial, les regalia occupent cependant une position singulière dans l'histoire de l'institution impériale. D'origine mythologique, ce sont des objets cachés qui n'ont jamais été montrés en public et que les empereurs eux-mêmes n'ont jamais vus, constitutifs à la fois d'un patrimoine matériel réel puisque transmissibles, mais invisibles. Ils échappent ainsi non seulement aux procédures habituelles de classement pour assurer leur conservation mais aussi à leur exhibition publique et leur valeur ne dépend ni de leur authenticité - contestée - ni de leur fabrication, ni de leur composition - inconnues -, mais de l'aura de mystère qui les enveloppe et les soustrait à toute tentative d'évaluation patrimoniale.

The Mirror, the Jewel and the Sword are traditionally viewed as the symbols of the Japanese monarchy handed down to successive generations of emperors. Having long been associated with the fundamental virtues ascribed to the throne, the imperial regalia occupy a peculiar status in the history of the Japanese imperial institution. Shrouded in their mythological origins, the three treasures that the emperors themselves were never allowed to see, have never been exposed in public. Nevertheless, they are a part of a material but invisible heritage, that has to be 
transmitted. Not only do they escape the current procedures of classification for their protection and any kind of public exhibition, but their value also lies neither in their so-called authenticity nor in their manufacturing or composition- which are unknown- but only in the aura of mystery which surrounds them and still impedes any attempt of heritage evaluation.

\section{INDEX}

Keywords : imperial regalia, Japan, monarchy, movable heritage, artefacts, status, Imperial Household Agency

Mots-clés : regalia, Japon, monarchie, patrimoine mobilier, objets d'art, statut, agence de la Maison impériale

\section{AUTEUR}

\section{ÉRIC SEIZELET}

Professeur émérite en langue et civilisation japonaises à l'université Paris-Diderot, ancien directeur de l'Institut d'Asie orientale de l'université Lumière-Lyon, directeur de recherche au CNRS et professeur à l'Inalco.

eseizelet1@gmail.com 